\title{
Die „Verkehrsfähigkeit von Beweisen“ im Strafverfahren
}

Von Dr. Sabine Gleß, Wiss. Referentin am Max-Planck-Institut für ausländisches und internationales Strafrecht, Freiburg i. Br.

Im Dezember 2001 hat die Kommission der Europäischen Gemeinschaften (EG) ein „Grünbuch zum strafrechtlichen Schutz der finanziellen Interessen der Europäischen Gemeinschaften und zur Schaffung einer Europäischen Staatsanwaltschaft " 1 vorgelegt. In diesem Diskussionspapier entwickelt sie ihr Konzept für eine supranationale EG-Strafverfolgungsbehörde. Dazu gehören auch Regelungsvorschläge für die Übermittlung strafprozessualer Beweismittel von einem Mitgliedstaat in einen anderen. Der Inhalt dieser Vorschläge und der ausdrückliche Wunsch der Kommission nach einer öffentlichen Dis kussion geben Anlass, zu diesem Thema Stellung zu nehmen ${ }^{2}$.

Die Konsequenz einer Umsetzung der im Grünbuch unterbreiteten Vorschriften für einen grenzüberschreitenden Beweistransfer wäre die Institutionalisierung eines „europaweit verkehrsfähigen Beweises " im Strafverfahren $^{3}$. Dieses Konzept entspricht den rechtspolitischen Forderungen nach einer verstärkten gegenseitigen Anerkennung strafprozessualer Maßnahmen und Entscheidungen in der EU, die - neben der EG-Kommission - auch von den Regierungen der EU-Mitgliedstaaten vorgebracht werden ${ }^{4}$ und die sich eng an die anerkannte Dogmatik des Europäischen Gemeinschaftsrechts im Bereich der wirtschaftlichen Zusammenarbeit anlehnen. Eine Analyse des Vorschlags zeigt aber, dass das vorgelegte Konzept für einen curopaweit verwertbaren strafprozessualen Beweis die grundlegende - in allen nationalen Rechtsordnungen gleiche - Funktion des Beweises im Strafverfahren nicht

1 Grünbuch zum strafrechtlichen Schutz der finanziellen Interessen der Europäischen Gemeinschaften und zur Schaffung einer Europäischen Staatsanwaltschaft, KOM (2001) 715 endgültig, im Folgenden: Grünbuch.

2 Stellungnahmen können unter <http://www.europa.eu.int/comm/ati_fraud/green paper/index_en.html> abgerufen werden; siehe auch Braum, ZRP 2002, 508; Brïner/ Spitzer, NStZ 2002, 393; Scbünemann, GA 2002, 510; Vogel, GA 2002, 533.

3 Vgl. dazu auch Art. 8 Abs. 3 VO (Euratom, EG) Nr. 2185/96 des Rates vom 11.11.1996 betreffend die („administrativen“) Kontrollen und Überprüfungen vor Ort durch die Kommission pp. (ABl. Nr. L 292 v. 15.11.1996, 2) sowic die Literaturnachweise in Anm. 8.

4 Vgl. etwa die Schlussfolgerungen des EU-Vorsitzes von Tampere (15./16.10.1999) oder die Initiative Frankreichs, Schwedens und Belgiens für einen Rahmenbeschluss über die Vollstreckung von Entscheidungen über die Sicherstellung von Vermögensgegenständen oder Beweismitteln in der EU (AB1. Nr. C 75 v. 7.3.2001, 3). 
gewährleisten kann: nämlich als Substrat für eine zuverlässige und faire Feststellung von (vergangenen) Tatsachen zu dienen, auf deren Grundlage das erkennende Gericht eine strafrechtliche Entscheidung treffen kann ${ }^{5}$.

\section{I. „Beweistransfer“ in der Europäischen Union (EU)}

Die Frage der Zulässigkeit bzw. der Voraussetzungen eines Beweistransfers, also einer Übermittlung von Beweismitteln von einer Rechtsordnung in eine andere, hat in den vergangenen Jahren vermehrt Interesse in der strafrechtswissenschaftlichen Literatur erfahren ${ }^{6}$. Anlass für die Diskussionen gibt vor allem der Ausbau und die Intensivierung der Zusammenarbeit Zwischen den EU-Mitgliedstaaten im Bereich der Strafverfolgung. Dieser Ausbau erfolgte auf ganz unterschiedlichen Ebenen: Die Mitgliedstaaten haben nicht nur die sonstige (traditionelle) Rechtshilfe - inklusive der grenzüberschreitenden Beweisaufnahme - durch das EU-Rechtshilfeübereinkommen vom Mai 2000 entscheidend vereinfacht, sondern auch für die lokalen Behörden neue Erkenntnismöglichkeiten (etwa durch die Schengen-Zusammenarbeit) ausgebaut $^{7}$. Im Rahmen der sog. 3. Säule der EU erleichtern das Europäische Polizeiamt (Europol) sowie die justitielle Kooperationsstelle Euro-Just den Informationsaustausch. Im Bereich des Gemeinschaftsrechts sollen „Kontrollberichte“ des Europäischen Amts für Betrugsbekämpfung (OLAF) „in der gleichen Weise und unter denselben Bedingungen wie die Verwaltungsberichte der Kontrolleure der einzelstaatlichen Verwaltungen Beweismittel dar[stellen], die in den Verwaltungs- oder Gerichtsverfahren des Mitgliedstaats, in dem sich ihre Verwendung als erforderlich erweist, zulässig sind. Für sie gelten dieselben Beurteilungsmaßstäbe wie für die Verwaltungs-

5 Dazu etwa für das deutsche Verfahren $E b$. Schmidt, Lehrkommentar zu StPO und GVG, Bd. I, Rdn. 296; Eisenberg, Beweisrecht, 4. Aufl. 2002, Rdn. 1 f., 1050 ff.; Krauß, Festschrift für Schaffsteịn, 1975, S. $411 \mathrm{ff}$; für Frankreich Rassat, Traité de Procédure Pénale, Paris 2001, no. 191ff.; für England Andrews and Hirst on Criminal Evidence, 3. Aufl. London 1997, 1-001; Seabrooke/Sprack, Criminal Evidence \& Procedure, Gosport 1999, S. 1f. Aus rechtsvergleichender Perspektive Pradel, Droit pénal comparé, Paris 1995, no. 271; aus rechtssoziologischer Sicht Lüderssen, ZStW 85 (1973), S. 291; Lubmann, Legitimation durch Verfahren, 1983, S. $60 \mathrm{ff}$.

6 Allgemein zur Problematik der Verwertung von „Auslandsbeweisen“ Böse, ZStW 114 (2002), S. 148; Körner, BtMG, 4. Aufl. 1994, $\$ 31$ Rdn. 179; Lagodny, in: Schomburg/ Lagodny, IRG, 3. Aufl. 1998, vor $\$ 59$ Rdn. 5, 5 a; Rose, Der Auslandszeuge im Beweisrecht des deutschen Strafprozesses, S. 158ff.; Walther, StV 1992, 561; Wilkitzki, in: Grützner/Pötz, IRG (Stand November 1991), vor $\$ 68 \mathrm{Rdn} .18$.

7 Dazu etwa Gleß, NStZ 2000, 57. 
berichte der einzelstaatlichen Verwaltungskontrolleure, und sie sind in Bezug auf diese als gleichwertig zu betrachten. ${ }^{\text { } 8}$

\section{Problematik}

Vor diesem Hintergrund der immer enger werdenden Zusammenarbeit der Strafverfolgungsbehörden und der Gerichte der EU-Mitgliedstaaten einerseits und eigener Ermittlungstätigkeit supra-nationaler Organe der EG andererseits stellen sich in Rechtswissenschaft und -praxis verschiedene Fragen, die über das Grundkonzept des Grünbuchs hinausgehen: Unter welchen Voraussetzungen und in welchem Umfang sollen

(1.) im Rahmen eines nationalen Verfahrens erhobene Beweise in den anderen Mitgliedstaaten,

(2.) in Zusammenhang mit (durch nationale Stellen durchgeführten) grenzüberschreitenden Ermittlungsmaßnahmen gewonnene Erkenntnisse in den jeweiligen Mitgliedstaaten und

(3.) Ergebnisse aus Untersuchungen der EG-Organe in allen Mitgliedstaaten

verwertbar sein?

Während sich die erste Frage mit echtem Beweistransfer im traditionellen Sinne befasst, könnte der zweite Komplex als neuartige „transnationale Beweissammlung " 9 bezeichnet werden und der dritte als - jetzt erst im Entstehen befindliche - „supranationale Beweissammlung “10. Das Konzept des Grünbuchs kann (und soll wohl künftig) für alle drei Komplexe nutzbar gemacht werden. Ausgerichtet ist es zunächst aber auf den erstgenannten Bereich.

8 Art. 8 (3) Satz 3 und 4 der VO Nr. 2185/96 (Anm. 3). Dazu Dannecker, ZStW 111 (1999), S. 293; Jokisch, Gemeinschaftsrecht und Strafverfahren, 1999, S. 245; Nelles, ZStW 109 (1997), S. 745; Sieber, ZRP 2000, 191.

9 Diese Form der Beweissammlung umfasst eigenständige Ermittlungen durch Hoheitsträger eines Staates auf dem Territorium eines anderen Staates (wie etwa bei der Schengen-Zusammenarbeit; Art. 40, 41 SDÜ) oder den Einsatz im Rahmen gemeinsamer Ermittlungsteams, deren Erkenntnisse allen Beteiligten zur Verfügung gestellt werden (vgl. Art. 13 EU-RhÜbk v. 29.5.2000, ABl. C 197 v. 12.7.2000, 1).

10 Bereits heute erfolgt durch das Europäische Amt für Betrugsbekämpfung (OLAF) eine "supranationale Beweissammlung“ im Rahmen „administrativer Untersuchungen“; vgl. oben Anm. 8. Dazu Vogel, in: Grützner/Pötz, IRG (Stand Dezember 2001) vor $\$ 1$, Rdn. 179. Auf eine „supranationale Bewcissammlung“ sind auch die Konzepte für cine Europäische Staatasanwaltschaft langfristig ausgerichtet; vgl. hierzu das Grünbuch (Anm. 1) einerseits und die Vorschläge des "Corpus Juris“-Entwurfes (Anm. 24) andererseits. 
2. Lösungswege de lege lata und de lege ferenda

In diesem ersten - bisher durch die klassische Rechtshilfe geregelten - Bereich gibt bereits heute die Rechtsprechung zur Verwertung von Auslandsbeweisen Antworten. In Auseinandersetzung mit dieser Rechtsprechung hat auch die Literatur verschiedene Reformvorschläge entwickelt.

\section{a) Rechtsprechung}

Die deutsche Rechtsprechung löst die Probleme des Beweistransfers - de lege lata - in einer pragmatischen Herangehensweise: Auslandsbeweise werden grundsätzlich auch dann für die Tatsachenfeststellung vor Gericht zugelassen, wenn ihre Sammlung bzw. Erhebung nicht den deutschen Verfahrenserfordernissen entspricht ${ }^{11}$. Die Nichtbeachtung deutscher Verfahrensvorschriften soll dann aber gegebenenfalls im Rahmen der Beweiswürdigung berücksichtigt werden ${ }^{12}$. Das bedeutet etwa, dass ein im Ausland gefertigtes Protokoll über eine Vernehmung, die - entsprechend der Verfahrensordnung des fremden ersuchten Staates - nicht durch einen Richter bzw. ohne Benachrichtigung und Anwesenheit der Verteidigung durchgeführt wurde, gleichwohl in Deutschland als "richterliches Protokoll “ nach $\$ 251$ Abs. 1 StPO verlesen werden darf ${ }^{13}$. Erst im Rahmen der Beweiswürdigung gleicht der Richter die (aus deutscher Sicht vorliegenden) Mängel in der Beweiserhebung aus ${ }^{14}$. Lediglich in Ausnahmefällen zieht die Nichteinhaltung von Verfahrensvorschriften des ersuchenden Staates, dessen Gerichte das Beweismittel verwerten wollen, den gänzlichen Ausschluss des Beweismittels nach sich ${ }^{15}$. Diese Judikatur führt - wie bereits von verschiedener Seite zutreffend bemängelt wurde - zu einer (unerwünschten) Ent-Förmlichung des Beweisverfahrens im nationalen Strafverfahren, da Umstände, die auf der (strenger formalisierten) Ebene der Beweiszulassung zu einem Beweis-

11 BGHSt. 2, 300, 304; BGH GA 1964, 176; 1976, 218, 219; BGH NStZ 1985, 376.

12 BGHSt. 2, 300, 304; OLG Hamm DAR 1959, 192, 193; Kohlhaas, NJW 1954, 535.

13 Vgl. BGH NStZ 1994, 595 m. w. N.; OLG Celle StV 1995, 179; Meyer-Goßner, StPO, 46. Aufl. 2003, $\$ 168$ c Rdn. 8, $\$ 251$ Rdn. 20f.; Scblücbter, in: SK StPO, $\$ 251$ Rdn. 37. Nach Wilkitzki, in: Grützner/Pötz (Anm. 6), vor $\$ 68 \mathrm{Rdn}$. 18, müssen jedenfalls die Verteidigungsrechte in dem durch Art. 6 Abs. 3 lit. d EMRK und Art. 10 des Internationalen Paktes über Bürgerliche und Politische Rechte festgelegten Mindestmaß gewährt werden.

14 BGHSt. 2, 300, 304; OLG Hamm DAR 1959, 192, 193; Koblhaas, NJW 1954, 535.

15 Dazu etwa Böse, ZStW 114 (2002), S. 153; Gleß, Festschrift für Grünwald, 1999, S. 197, 204. 
ausschluss führen müssten, erst auf der Ebene der Beweiswürdigung berücksichtigt werden können ${ }^{16}$.

\section{b) Reformvorschläge}

Die Kritik an der pragmatischen Herangehensweise der Judikatur war auch der erste Anstoß für eine Reformdiskussion ${ }^{17}$. Gesucht wurden neue Wege zur Verwertung von Auslandsbeweisen, die auch im Falle eines Beweistransfers sicherstellen, dass eine - aus Sicht der Rechtsgemeinschaft - zuverlässige und faire Herstellung der Tatsachengrundlage im Strafverfahren gewährleistet ist.

Zunächst beschränkten sich die aufgezeigten Lösungsmöglichkeiten nur auf Einzelfallkorrekturen ${ }^{18}$, später folgten Forderungen nach einem „Paradigmenwechsel“ im Rechtshilferecht ${ }^{19}$. Einen grundsätzlich neuen Ansatz verfolgt etwa das EU-Rechtshilfeübereinkommen vom 29.5.2000 (EURhÜbk) zur Lösung der Probleme des klassischen Beweistransfers ${ }^{20}$ : Nach Art. 4 EU-RhÜbk soll im Rechtshilfeverkehr zwischen den EU-Mitgliedstaaten künftig der Grundsatz „forum regit actum“ den traditionellen Grundsatz „locus regit actum“ ersetzen ${ }^{21}$. Das heißt, dass sich die Erledigung von Rechtshilfeersuchen nicht mehr nach dem Verfahrensrecht des ersuchten, sondern nach dem des ersuchenden Staates richten wird ${ }^{22}$. Parallel dazu normiert das EU-RhÜbk gemeinsame Verfahrensregeln, etwa für die (grenzüberschreitende) Telefonüberwachung ${ }^{23}$. Die Vorschläge des „Corpus Juris“-Entwurfes ${ }^{24}$ sind demgegenüber bereits stärker auf eine trans-

16 Etwa Perron, ZStW 112 (2000), S. 208; Vogel, ZStW 110 (1998), S. 977; vgl. auch Renzikowski, JZ 1999, 610.

17 Vgl. etwa Thien, Zeugenvernehmung im Ausland, Diss. Köln 1979; Walther, StV 1992, $562 \mathrm{f}$.

18 Wie etwa bei Thien (Anm. 17); Scheller, Ermächtigungsgrundlagen für die internationale Rechts- und Amtshilfe zur Verbrechensbekämpfung, 1997, S. $279 \mathrm{ff}$.

19 Vgl. Perron, ZStW 112 (2000), S. 208, und Vogel, ZStW 110 (1998), S. 977, die eine grundsätzliche Anwendung des „forum regit actum"-Prinzips fordern, oder Böse, ZStW 114 (2002), S. $156 \mathrm{ff}$., der durch neue „Steuerungsmöglichkeiten “ bei der inländischen Verwertung ausländischer Beweismittel die informationellen Schutz- und Abwehrrechte des einzelnen ausreichend schützen möchte.

20 Übereinkommen über die Rechtshilfe in Strafsachen zwischen den Mitgliedstaaten der Europäischen Union (EU-RhÜbk), ABl. EG Nr. C 251 v. 2.9.1999, 1.

$21 \mathrm{Zu}$ diesen Rechtshilfegrundsätzen ausführlich Gleß, Festschrift für Grünwald, S. 197 ff.; Nagel, Beweisaufnahme im Ausland, 1988, S. 161 ff., m. w. N

22 Dazu Lagodny, Festschrift für Spinellis, 2002, S. 641.

23 Vgl. Art. $17 \mathrm{ff}$. EU-RhÜbk.

24 Delmas-Marty, Corpus Juris der strafrechtlichen Regelungen zum Schutz der finan- 
bzw. supranationale Beweiserhebung ausgerichtet. Er sieht in Art. 32 die Einführung bestimmter originärer „europäischer Beweismittel“ vor, die in allen nationalen Verfahren verwertet werden können ${ }^{25}$; gegebenenfalls müssen sie durch den "Richter im Ermittlungsverfahren “ ${ }^{\text {" }}$ ugelassen werden ${ }^{26}$. Getragen wird dieser Vorschlag von der Überzeugung, dass durch die Geltung der EMRK in den einzelnen Rechtsordnungen ein gemeinsamer Standard für europaweit verwertbare Beweismittel besteht ${ }^{27}$.

Im Zentrum der bisherigen Lösungsvorschläge stehen vorwiegend Überlegungen zu einer besseren verfahrensrechtlichen und institutionellen Verankerung des Beweistransfers in Europa. Es fehlen aber weitgehend grundlegende Überlegungen dazu, ob bzw. wann ein (bestimmtes) Beweismittel, das unter der Geltung der Rechtsordnung eines Mitgliedstaates der EU mit dem Ziel der Verwertung in dem zu dieser Rechtsordnung gehörenden Strafverfahren erhoben wurde, überhaupt ein taugliches Beweismittel unter der Geltung einer anderen EU-Rechtsordnung sein kann. Gemeinsame Prämisse der bisherigen Diskussion scheint vielmehr zu sein, dass strafprozessuale Beweise grundsätzlich dem Konzept der gegenseitigen Anerkennung unterstellt werden können.

\section{Konzept eines „europaweit verkehrsfähigen Beweises“}

Der im Grünbuch ausgearbeitete Vorschlag setzt diese Prämisse am konsequentesten um ${ }^{28}$ : Das Grünbuch schlägt vor, dass mitgliedstaatliche Gerichte, die mit einem Strafverfahren befasst sind, dem eine (mutmaßliche) Beeinträchtigung der finanziellen Interessen der EG zugrunde liegt, verpflichtet werden, jedes Beweismittel zuzulassen, das nach dem nationalen Recht eines anderen Mitgliedstaats rechtmäßig erhoben wurde ${ }^{29}$.

ziellen Interessen der Europäischen Union, 1998, S. 98f. Ein überarbeiteter Entwurf ("Fassung von Florenz") ist abrufbar unter <http://www.law.uu.nl/wiarda/corpus/ index1.htm>.

25 Zur Evaluation dieser Vorschrift durch die Autorengruppe selbst Delmas-Marty/Vervaele, The Implementation of the Corpus Juris in the Member States, Antwerpen 2000, Bd. I, S, 98f., m. w.N.

26 Art. 25 Abs. 3 Corpus Juris.

27 Vgl. die Beweisverwertungsregelung in Art. 33 Abs. 1 Corpus Juris; dazu Nelles, in: Huber (Hrsg.), Das Corpus Juris als Grundlage eines Europäischen Strafrechts, 2000, S. $271 \mathrm{f}$.

28 Vgl. dazu unten II. 2. a. sowie Brïner/Hetzer, NStZ 2003, 113.

29 Grünbuch (Anm. 1), 6.3.4.1. 
1. (Gemeinschaftsrechtlicher) Hintergrund des Konzepts eines „europaweit verkehrsfähigen Beweises“

Das im Grünbuch angelegte Konzept für eine „Verkehrsfähigkeit“ von strafprozessualen Beweismitteln ${ }^{30}$ intendiert damit - anders als etwa das EURechtshilfeübereinkommen vom Mai 2000 - nicht (nur) eine Vereinfachung der Rechtshilfe, sondern deren Ersetzung durch einen „freizügigen Beweis“. Als Grund für diesen - dem klassischen Rechtshilferecht diametral entgegengesetzten - Richtungswechsel verweisen die Autoren des Grünbuchs auf die Schwerfälligkeit des traditionellen Rechtshilfeverkehrs ${ }^{31}$. Eine Vereinheitlichung durch das neue Modell wird aus ihrer Sicht dadurch gerechtfertigt, dass sich in den EU-Mitgliedstaaten durch die Geltung der EMRK ein gemeinsamer Mindeststandard herausgebildet habe, dessen Einhaltung in den einzelnen Rechtsordnungen eine grenzüberschreitende Verwertung von rechtmäßig gesammelten Beweismitteln erlaube ${ }^{32}$. Eine vergleichbare Argumentation findet sich bereits im "Corpus Juris“-Entwurf ${ }^{33}$.

Das hinter dem Grünbuch-Modell stehende Konzept lehnt sich offensichtlich an das „Prinzip der Freizügigkeit“ an, das im europäischen Gemeinschaftsrecht mit dem Ziel der Herstellung eines EG-Binnenmarktes entwickelt wurde: Die gemeinschaftsrechtliche Doktrin basiert auf der Prämisse, dass (a) „Produktions"-Vorschriften in einem Staat grundsätzlich adäquat zu den „Produktions"-Vorschriften aller anderen Staaten sind und dass (b) diese Voraussetzung genügt, damit ein einmal erzeugtes Produkt auch unter der Geltung anderer Rechtsordnungen verbraucht werden kann ${ }^{34}$. Das (fertige) Produkt, für das Freizügigkeit geltend gemacht wird, wird also so behandelt, als ob es im eigenen Staate, unter der Geltung der

30 Vergleichbare Ansätze gab es bereits davor. Art. 10 Abs. 4 des Entwurfs für ein Zusatzprotokoll zu dem Übereinkommen über den Schutz der finanziellen Interessen der EG v. 19.1.1996 (ABl. C 83 v. 20.3.1996, 10) sah vor, dass „[d]ie in einem Mitgliedstaat amtlich festgestellten Tatumstände ... in allen anderen Mitgliedstaaten gültige Beweismittel [sind], wie wenn sie nach dem innerstaatlichen Recht erstellt worden wären"; eine vergleichbare Regelung für „administrative“ Betrugsermittlungen wurde in Art. 8 Abs. 3 VO 2185/96 (Anm. 8) geschaffen.

31 Vgl. Grünbuch (Anm. 1) 6.2.2. Den empirischen Beleg für diese Behauptung bleibt das Grünbuch allerdings schuldig. Dieser Mangel wurde insbesondere von den Mitgliedstaaten in ihren Stellungnahmen (Anm. 2) kritisch angemerkt; vgl. auch Fijnaut/Groenbuijsen, European Journal of Crime, Criminal Law and Criminal Justice 11 (2003), S. 327.

32 Vgl. Grünbuch (Anm. 1), 6.2.1.1

33 Vgl. Art. 25 Abs. 2 Corpus Juris.

34 Vgl. etwa Streinz, Europarecht, 5. Aufl. 2001, Rdn. 657, 955ff.; Schreeitzer/Hummer, Europarecht, 5. Aufl. 1996, Rdn. $1062 \mathrm{ff.}$ 
eigenen Rechtsordnung produziert worden wäre. Entsprechend diesem Prinzip sollen Beweismittel, die in einem Mitgliedstaat rechtmäßig erhoben - und damit „produziert ${ }^{c}$ - wurden, in allen anderen Mitgliedstaaten für die Feststellung der Tatsachengrundlage durch ein Strafgericht zugelassen werden. In concreto müsste der Vorschlag der EG-Kommission also bedeuten, dass ein Beweismittel, das in einem EG-Mitgliedstaat rechtmäßig erhoben wurde, in allen anderen Mitgliedstaaten obne weitere Prüfung vor Gericht als Beweismittel zugelassen wird - mit der Fiktion, dass dieses Beweismittel (rechtmäßig) nach der eigenen Verfahrensordnung des erkennenden Gerichts erlangt wurde ${ }^{35}$. Die Filterfunktion, die bisher im Rahmen der traditionellen Rechtshilfe durch die Beweiszulassung bzw. die Beweiswürdigung im nationalen Verfahren erfolgte, dürfte damit entfallen.

2. Übertragbarkeit des gemeinschaftsrechtlichen Konzepts der „Verkehrsfähigkeit“ auf strafprozessuale Beweise?

$\mathrm{Zu}$ prüfen ist erstens, welche konkrete Bedeutung das im Grünbuch vorgeschlagene Modell für den Austausch strafprozessualer Beweise haben soll, und zweitens, ob das von der EG-Kommission vorgelegte Konzept geeignet ist, die mit einem Beweistransfer verbundenen Probleme zu lösen. Eine Problemlösung liegt nur dann vor, wenn das vom Grünbuch als maßgeblich anerkannte Kriterium („rechtmäßige Beweisgewinnung in einem Mitgliedstaat") sicherstellen kann, dass ein unter der Geltung einer Rechtsordnung gewonnenes „Beweismittel“ unter der Geltung einer anderen Rechtsordnung die Funktion eines Beweises im Erkenntnisverfahren übernehmen kann.

\section{a) Konkreter Bedeutungsgehalt des Grünbuch-Modells}

Das Grünbuch schlägt vor, dass mitgliedstaatliche Gerichte, die mit einem Strafverfahren befasst sind, dem eine (mutmaßliche) Beeinträchtigung der finanziellen Interessen der EG zugrunde liegt, verpflichtet werden, jedes Beweismittel zuzulassen, das nach dem nationalen Recht eines anderen Mitgliedstaats rechtmäßig erhoben wurde ${ }^{36}$. Den konkreten Bedeutungsgehalt dieser Regelung lässt das Grünbuch aber offen.

35 So auch Fijnaut/Groenbwijsen, European Journal of Crime, Criminal Law and Criminal Justice 11 (2003), S. 333 f.; vgl. aber die restriktive Interpretation einer „europaweiten Gleichstellung" durch Spencer, in: Delmas-Marty/Vervaele, S. 242.

36 Grünbuch (Anm. 1), 6.3.4.1. 


\section{aa) „Berweise zulassen"}

So stellt sich etwa die Frage, ob nach diesem Modell „fertige Beweismittel“ grenzüberschreitend übermittelt werden sollen, mit der Konsequenz, dass sie durch die rechtmäßige Erhebung in einem Mitgliedstaat in einer Beweisform gebunden sind (etwa als zulässiger Urkunds- oder Zeugenbeweis), oder ob lediglich Resultate von (quasi abgetrennt im Ausland durchgeführten) Beweisaufnahmen übermittelt werden sollen, die anschließend in dem verwertenden Land noch einer bestimmten Beweismittelform zugeordnet werden können und müssen. Letzteres hätte zur Folge, dass im Ausland gewonnene Informationen nur entsprechend den Regeln der Rechtsordnung des verwertenden Gerichts als zulässiger strafprozessualer „Beweis“ präsentiert werden können.

Die Bedeutung dieser Frage lässt sich an folgendem Fall illustrieren: In Frankreich, Deutschland und England ${ }^{37}$ werden parallel gegen verschiedene Personen Ermittlungen wegen des Verdachts einer strafbaren Verwendung von EG-Subventionen geführt. Im Rahmen einer französischen gerichtlichen Voruntersuchung wird ein Zeuge vernommen und ein entsprechendes Protokoll für die Hauptverhandlung angefertigt ${ }^{38}$. Nach französischem Verständnis liegt damit ein rechtmäßig erhobener Zeugenbeweis vor, der über das Protokoll (als „preuve littérale") in das Hauptverfahren eingeführt werden darf ${ }^{39}$. Nach französischem Verfahrensrecht wäre eine nochmalige Vernehmung des Zeugen in der Hauptverhandlung zwar durchaus möglich ${ }^{40}$ und in bestimmten Fällen wohl auch üblich, aber nicht unbedingt notwendig ${ }^{41}$.

Fraglich ist, ob nach dem im Grünbuch-Modell diese in Frankreich rechtmäßig erhobene „preuve littérale“ ohne weiteres in die englische

37 England und Wales haben eine gemeinsame Strafrechtsordnung; soweit im Folgenden von England gesprochen wird, ist damit auch Wales gemeint.

38 Art. 102 und Art. 106 Code de procédure pénale (CPP). Zur Zeugenvernehmung in der französischen gerichtlichen Voruntersuchung Rassat, Traité de procédure pénale, no. 253; rechtsvergleichend zur Verwertung von polizeilichen Vernehmungsprotokollen im Hauptverfahren Meyer, ZStW 105 (1993), S. 386.

39 Vgl. Art. 310 CPP; Conte/Maistre du Chambon, Procédure pénale, 3. Aufl., no. 50.

40 Vgl. Art. 331, 444, 536 CPP; zur Beweismittelfreiheit („principe de la liberté de la preuve $^{\text {") }}$ auch für den Nachweis der Schuld Levasseur/Chavanne/Montrewil/Bouloc, Droit pénal général et procédure pénale, 1999, Rdn. 438; Pradel, Procédure pénale, Rdn. 275; Stefani, Encyclopédie Dalloz, „Preuve“, Rdn. 23.

41 Zur Tatsachenfeststellung durch die Würdigung der im Vorverfahren festgestellten Beweise Crim. 1. avr. 1992, B. no. 137; Conte/Maistre du Chambon (Anm. 39), no. 537. Zur Beweisfeststellung im Vorverfahren und deren Würdigung im Hauptverfahren Pradel (Anm. 40), Rdn. 549. 
Hauptverhandlung eingeführt werden kann und muss (weil sie bereits in dieser Form als zulässiger Beweis feststeht) oder ob der englische Richter zunächst prüfen kann und muss, ob die Verwertung der ursprünglich aus der Zeugenaussage gewonnenen Informationen der "hearsay rule unterfällt und deshalb nur verwertet werden darf, wenn sie durch einen Ausnahmetatbestand zugelassen wird (weil eben nur das Resultat der in Frankreich durchgeführten Beweissammlung übermittelt wird) ${ }^{42}$. In letzterem Fall wäre eine Verwertung wegen der englischen „hearsay rule" problematisch, die besagt, dass das erkennende Gericht Informationen über Tatsachen, die durch eine Person wahrgenommen wurden, nur dann für die Sachverhaltsfeststellung berücksichtigen darf, wenn diese Person selbst vor dem entscheidenden Gericht darüber berichtet ${ }^{43}$. Sie sichert damit den traditionell stark ausgeprägten Unmittelbarkeits- und Mündlichkeitsgrundsatz des angelsächsischen Strafverfahrens ab und soll garantieren, dass grundsätzlich nur solche Zeugenaussagen zugelassen werden, deren Zuverlässigkeit durch Kreuzverhör und Eid abgesichert werden kann ${ }^{44}$ bzw. deren Informationsgehalt nicht durch die Wiedergabe einer dritten Person verfälscht worden sein kann ${ }^{45}$. Das französische Strafverfahrensrecht geht demgegenüber davon aus, dass die Wahrnehmung eines Augenzeugen regelmäßig dadurch zu einer zuverlässigen Grundlage für eine Sachverhaltsfeststellung durch das erkennende Gericht wird, dass ein zeitlich kurz nach der Tat durch ein offizielles Organ aufgenommenes Protokoll in das Strafverfahren eingeführt wird. Dahinter steht der Gedanke, dass dieses professionell angefertigte Schriftstück, das unter dem frischen Eindruck des Wahrgenommenen entsteht, dem erkennenden Gericht bessere Rückschlüsse auf das Vorliegen einer Tatsache in der Vergangenheit erlaubt ${ }^{46}$.

42 Vgl. dazu etwa Spencer, in: Delmas-Marty/Vervaele (Anm. 25), S. 242, einerseits, Fijnaut/Groenbuijsen, European Journal of Crime, Criminal Law and Criminal Justice 11 (2003), S. $333 \mathrm{f}$. , andererseits.

43 "An assertion other than one made by a person while giving oral evidence in the proceedings is inadmissible as evidence of any fact asserted. “; R. v. Sharp (1988) 1 WCR 7 , (1988) 1 All ER 67.

44 Vgl. Teper v. R. (1952) AC 480, (1952) 2 All ER 447; Walker/Ward, English Legal System, 8. Aufl., S. 617; Zuckerman, Principles of Criminal Evidence, Oxford 1989, S. 179f. Zur "hearsay rule" und ihrer Anwendung im englischen Rechtskreis vgl. etwa Myers v. Director of Public Prosecution, (1965) AC 1001, (1964) 2 All ER 881; R. v. Karley, (1992) AC 228, (1992) 2 All ER 345; R. v. Rice, (1963) 1 All ER 332.

45 Osborne, Criminal Litigation, London 1998, S. 255.

46 Vgl. Conte/Maistre du Chambon (Anm. 39), no. 50f. Zur Grundproblematik vgl. Jung, GA 2002, 69. 
Für eine Auslegung in dem Sinne, dass lediglich das Resultat einer - quasi abgetrennt im Ausland durchgeführten - Beweisaufnahme übermittelt und zugelassen werden muss, könnte angeführt werden, dass das Grünbuch (nur) eine Verpflichtung zur Zulassung eines Beweismittels, nicht zu dessen Verwertung statuiert. Gegen ein solches Verständnis streitet aber das Ziel des Grünbuchs, nämlich einen möglichst reibungslosen Beweistransfer zwischen den Mitgliedstaaten zu garantieren. Stellt man dieses Ziel in den Mittelpunkt der Überlegungen, dann muss das im Grünbuch vorgelegte Konzept so interpretiert werden, dass nicht nur das Resultat einer (im Ausland durchgeführten) Beweisaufnahme als "Beweis" übermittelt werden soll, sondern bereits das „fertige Beweismittel“, das im verwertenden Land unabhängig von den dort im allgemeinen geltenden Vorschriften als Beweis präsentiert werden kann.

Wenn sich also - wie im Beispielsfall für den Zeugenbeweis - zwei unterschiedliche Konzepte einer Beweisform gegenüberstehen, die beide (auf verschiedene Weise) absichern wollen, dass aus der Präsentation von Informationen in der Gegenwart zuverlässige Rückschlüsse über das Vorliegen von Tatsachen in der Vergangenheit gezogen werden können, geht das Konzept des Erhebungsstaates vor. Dementsprechend dürfte der in Frankreich rechtmäßig erhobene Zeugenbeweis in das englische Erkenntnisverfahren eingeführt werden, ohne dass er den „hearsay“-Test durchlaufen muss.

\section{bb) Was ist ein (verkehrsfähiges) Beweismittel?}

Doch selbst wenn der konkrete Bedeutungsgehalt des Grünbuch-Modells insoweit geklärt ist, bleiben weitere grundlegende Fragen offen, wie etwa die nach den Charakteristika eines (verkehrsfähigen) Beweismittels. Das Grünbuch erläutert nicht, wann ein „Beweis“ vorliegt, sondern setzt ein feststehendes Konzept voraus. Ein solches Konzept gilt es aber zunächst herauszuarbeiten. Denn die Anerkennung einer Informationsquelle als Beweis für die strafprozessuale Tatsachenfeststellung existiert nicht als feststehender europäischer Begriff, sondern hängt u. a. von der Ausgestaltung der jeweiligen Rechtsordnung ab.

Zur Illustration soll der oben genannte Beispielsfall weitergeführt werden: In dem englischen Ermittlungsverfahren wird dem Beschuldigten $E$ von den Strafverfolgungsbehörden ein Vorschlag für einen „deal“ unterbreitet. Der Anwalt des E rät, das Angebot anzunehmen, da die Bedingungen gut seien und $\mathrm{E}$ nichts passieren könne, wenn er sich letztlich doch gegen den "deal"entscheide, denn ein in diesem Rahmen abgegebenes Schuldeinge- 
ständnis werde in einem gegebenenfalls anschließenden Strafverfahren nicht als Geständnis verwertet. Daraufhin erklärt sich $\mathrm{E}$ in einem Schreiben an die Behörden bereit zuzugeben, dass er EG-Subventionen veruntreut habe. Das Verfahren wird aber eingestellt, ohne dass es zum „deal“ kommt. Das Europäische Amt für Betrugsbekämpfung (OLAF) glaubt, das Verfahren sei zu Unrecht eingestellt worden. Es fordert von den englischen Behörden Kopien der Unterlagen über das Verfahren. Darin ist auch die Korrespondenz mit E (u. a. über die Möglichkeit eines ,guilty plea") dokumentiert. Diese übersendet OLAF an die deutschen Strafverfolgungsbehörden mit der Bitte, aufgrund dieser in England gesammelten „Beweismittel“ ein Verfahren gegen $\mathrm{E} z u$ eröffnen.

Gegenüber den deutschen Strafverfolgungsbehörden bestreitet $E$ den Vorwurf umfassend. Die deutschen Behörden möchten deshalb das von $\mathrm{E}$ in Vorbereitung eines „plea bargain" abgegebene „Schuldeingeständnis“ in das deutsche Verfahren einführen.

Das von der EG-Kommission vorgeschlagene Freizügigkeitsprinzip kann nur dann Anwendung finden, wenn ein „Beweis“ grenzüberschreitend transferiert werden soll. Fraglich ist, ob das zur Vorbereitung des englischen "guilty plea abgegebene "Schuldeingeständnis" ein in England rechtmäßig erhobenes „Beweismittel“ darstellt. Zweifel an der Beweismittelqualität des „Schuldeingeständnisses“ ergeben sich daraus, dass nach englischem Recht ein Beweisverfahren nur bei streitiger Verhandlung durchgeführt wird. Wenn die Parteien dem Strafverfahren einvernehmlich eine bestimmte Tatsachengrundlage zugrunde legen, entfällt die streitige Tatsachenfeststellung durch das Beweisverfahren ${ }^{47}$. Vor diesem Hintergrund geben Äußerungen des Beschuldigten im Rahmen der Vorbereitung eines „deal" also zunächst nur Auskunft darüber, auf welcher Grundlage der für das Verfahren relevante Sachverhalt - aus seiner Sicht - festgestellt werden könnte. Ein zu diesem Zweck angekündigtes bzw. abgegebenes Schuldeingeständnis wird nicht als Beweismittel erhoben. Nach englischem Verständnis verbietet sich eine Verwertung gegen den Angeklagten regelmäßig ${ }^{48}$, wobei der Ausschluss heute im Wesentlichen auf „Fairness"-Erwägungen zurückgeführt wird ${ }^{49}$.

47 Seabrooke/Sprack (Anm. 5), S. 6; Wolchover/Heaton-Armstrong, Confession Evidence, 1-004.

48 Blackstone's Criminal Practice, 2003, F17.1. Allerdings kannte bereits das „common law" Ausnahmen von diesem Grundsatz, wenn das Eingeständnis glaubhaft und eine Verwertung nicht unfair erschien; dazu Wolchover/Heaton-Armstrong (Anm. 47), $1-070 \mathrm{~m}$. w. N.

49 Dazu Wolchover/Heaton-Armstrong (Anm. 47), 1-070 m.w.N. 
Ein solches Ergebnis erscheint aber auch bei funktionaler Betrachtung des Schuldeingeständnisses aus der Perspektive des englischen Rechts gerechtfertigt, weil es sich bei diesem Schuldeingeständnis eben eher um eine Prozesserklärung als um ein Beweismittel handelt ${ }^{50}$.

Zweifelhaft ist, ob die deutsche Rechtsordnung überhaupt Mechanismen hätte, um eine solche Informationsquelle als (verkehrsfähiges) Beweismittel auszuschalten ${ }^{51}$. Denn die Dogmatik der deutschen Beweisverwertungsverbote ist am System der eigenen Verfahrensordnung ausgerichtet, die traditionell keine einverständliche Festlegung des Sachverhalts durch die Parteien kennt. Hier zeigt sich auch wieder die enge Verbundenheit von Beweisverboten und Grundprinzipien einer Strafverfahrensordnung.

\section{b) Tragfähigkeit des Grünbuch-Modells: Rechtmäßigkeit der Beweis- sammlung als maßgebliches Kriterium für die Zulässigkeit der Beweisverwertung}

$\mathrm{Zu}$ prüfen ist weiter, ob das vom Grünbuch als maßgeblich benannte Kriterium für die grenzüberschreitende Verwertbarkeit eines Beweismittels, nämlich seine rechtmäßige Gewinnung in einer mitgliedstaatlichen Rechtsordnung, eine tragfähige Lösung für die mit dem Beweistransfer verbundenen Probleme darstellt. Das ist (nur) dann der Fall, wenn ein unter Geltung einer Rechtsordnung rechtmäßig gesammeltes „Beweismittel“ auch in jeder anderen mitgliedstaatlichen Rechtsordnung die Funktion eines Beweises im Erkenntnisverfahren übernehmen kann, nämlich als Substrat für eine zuverlässige und faire Feststellung von (vergangenen) Tatsachen zu dienen.

aa) Zuverlässigkeit der Tatsachenfeststellung (aus Sicht der Rechtsgemeinschaft, in deren Namen die Sachentscheidung getroffen wird)

In den europäischen Rechtsordnungen bestehen ganz unterschiedliche Ansichten darüber, wie aus in der Gegenwart vorliegenden Informationen möglichst zuverlässig Rückschlüsse auf die Existenz von (strafrechtlich relevanten) Tatsachen in der Vergangenheit gezogen werden können.

Knüpft man an den oben geschilderten Fall der Zeugenvernehmung in der französischen gerichtlichen Voruntersuchung an, deren Ergebnis als "preuve littérale“ in das Hauptverfahren eingeführt werden soll ${ }^{52}$, so stellt

$50 \mathrm{Vgl}$. Weigend, ZStW 94 (1982), S. 201.

51 Vgl. BGHSt. 21, 285, 287; Kuckein, Festschrift für Meyer-Goßner, 2001, S. 66 m. w.N.

52 Dazu etwa Conte/Maistre du Chambon (Anm. 39), no. 50. 
sich die Frage, ob das französische Protokoll unter den Prämissen des englischen Erkenntnisverfahrens ein Beweismittel sein kann, das aus Sicht der dortigen Rechtsgemeinschaft eine zuverlässige (und faire) Sachverhaltsfeststellung gewährleisten kann. Nach englischem Verständnis und Verfahrensrecht gilt die mündliche Aussage des Zeugen vor dem erkennenden Gericht (wegen der Möglichkeit des Wechselverhörs, der „cross-examination“, und des Verbots der Wiedergabe durch dritte Personen) traditionell als Sicherung gegen unzuverlässige Aussagen ${ }^{53}$. „Hearsay " kann nur dann der Tatsachenfeststellung zugrunde gelegt werden, wenn ein Ausnahmetatbestand zur "hearsay rule " eingreift ${ }^{54}$. Was aus französischer Sicht also eine zuverlässige Grundlage für dic Sachverhaltsfeststellung gibt - das zeitlich kurz nach der Wahrnehmung professionell angefertigte Protokoll, selbst wenn es unter diesen Umständen nur in beschränktem Umfang einer „,kontradiktorischen Erörterung " zugänglich ist ${ }^{55}$ - entspricht grundsätzlich nicht den englischen Anforderungen an eine zuverlässige Sachverhaltsfeststellung. Der „vorweggenommenen richterlichen Beweisaufnahme" im französischen Verfahrensrecht fehlt ein funktionales Äquivalent im englischen Strafprozess.

Die Rechtmäßigkeit der Beweissammlung in einem Mitgliedstaat erscheint somit nicht als taugliches Kriterium für die Beurteilung einer grenzüberschreitenden Verwertbarkeit; vielmehr bemisst sich die Frage der Verwertbarkeit (auch) nach Bedingungen, die sich aus dem Erkenntnisverfahren selbst ergeben ${ }^{56}$. Die Einhaltung der Anforderungen an eine rechtmäßige Beweissammlung stellt damit nur eine (weder hinreichende noch regelmäßig notwendige) Bedingung dar, deren Vorliegen bei der Prüfung der Verwertbarkeit eines Beweismittels zu berücksichtigen ist. Denn welche Bedeutung der Rechtswidrigkeit einer Beweissammlung für die Frage der Verwertbarkeit zukommt, wird in den nationalen Rechtsordnungen ganz unterschied-

53 Vgl. dazu etwa Teper v. R., (1952) AC 480, (1952) 2 All ER 447; Myers v. Director of Public Prosecution, (1965) AC 1001, (1964) 2 All ER 881; R. v. Karley, (1992) AC 228 (1992) 2 All ER 345; R. v. Rice (1963), 1 All ER 332; Osborne (Anm. 45), S. 255; Walker/Ward (Anm. 44), S. 617; Zuckerman (Anm. 44), S. 179f. Siehe auch oben Anm. 43.

$54 \mathrm{Zu}$ den Ausnahmen von der "hearsay rule" vgl. Walker/Ward (Anm. 44), S. 617; Zuckerman (Anm. 44), S. 201-214.

55 Dieser Umstand wird in der französischen Literatur mit Blick auf Art. 6 Abs. 3 lit. d EMRK kontrovers diskutiert; vgl. etwa Pradel (Anm. 40), no. 548 a. E.; kritisch dazu Meyer, ZStW 105 (1993), S. 391.

56 Vgl. etwa Grünwald, Das Beweisrecht der Strafprozessordnung, 1993, S. 143, der herausgearbeitet hat, dass in bestimmten Konstellationen das Verwertungsverbot das Vorrangige und das Erhebungsverbot lediglich Folge des primären Verwertungsverbotes sein kann. 
lich beurteilt ${ }^{57}$. Einen zwingenden Ausschluss rechtswidrig erlangter Beweismittel sehen die Rechtsordnungen nur ausnahmsweise vor ${ }^{58}$. Vielmehr führt gerade die unterschicdliche theoretische und praktische Relevanz von Beweisverwertungsverboten den spezifischen Zusammenhang der Beweisregelungen untereinander und mit den Grundprinzipien des jeweiligen Prozesssystems vor Augen ${ }^{59}$.

In Bezug auf die vorliegende Fragestellung kann damit festgehalten werden, dass für die grenzüberschreitende Verwertbarkeit nicht nur darauf abgestellt werden müsste, ob ein Beweismittel unter der Geltung der Rechtsordnung des Erhebungsortes erboben, sondern ob es dort auch hätte verwertet werden dürfen. Es gilt dabei zu beachten, dass die spezifischen Anforderungen an die Beweisgewinnung in einer Rechtsordnung zu einem wesentlichen Teil aus der Antizipation der Beweisverwertung (einschließlich der Beweiswürdigung) in dieser Rechtsordnung resultieren - gerade daher rührt ja der (im Grünbuch ganz beherrschende) Fokus auf die Umstände der Beweissammlung für die Beurteilung der Frage der Verwertbarkeit ${ }^{60}$. Anschließend müsste in jedem Einzelfall geprüft werden, ob bzw. inwieweit die Einhaltung der Beweisgewinnungsvorschriften auch bei Verwertung des Beweismittels in einer anderen. Rechtsordnung von Bedeutung ist ${ }^{61}$.

57 Für Deutschland siehe etwa Wolter, in: SK StPO, vor $\$ 151$ Rdn. 194ff.; Dencker, Festschrift für Meyer-Goßner, 2001, S. 239; Rogall, JZ 1996, 944; Weigend, ZStW 113 (2001), S. $288 \mathrm{ff}$. Für England Crompton J. in R. v. Leatham (1861), 8 Cox CC 498, und Kuruma v. R. (1955) AC 197; bestätigt beispielsweise in R. v. Mason, (1988) 1 WCR 139. Vgl. auch Osborne (Anm. 45), S. 299 f., und Zuckerman (Anm. 44), S. 314, 349f., einerseits und die gesetzlichen Regelungen in ss. 76 und 78 Police and Criminal Evidence Act 1984 andererseits (dazu Wolchover/Heaton-Armstrong [Anm. 47], 4-036ff.).

58 Dazu aus rechtsvergleichender Sicht Spencer, in: Delmas-Marty/Spencer (Hrsg.), European Criminal Procedures, Cambridge 2002, S. $603 \mathrm{ff}$., sowie die Beiträge in: Huber/ Höpfel (Hrsg.), Beweisverbote in Ländern der EU und vergleichbaren Rechtsordnungen, 1999.

59 So sollen etwa in England die Beweiszulassungsverbote des common law die Unvoreingenommenheit der Laienjury schützen; Andrews and Hirst (Anm. 5), no. 2-036 m. w. N.; Seabrooke/Sprack (Anm. 5), S. 1.

60 Dieser Zusammenhang zwischen Beweissammlungs- und Beweisverwertungsvorschriften wird oft damit umschrieben, dass jede nationale Strafverfahrensordnung ein Gesamtsystem bilde; vgl. etwa die Gemeinsame Stellungnahme der Bundesregierung und der Länder der Bundesrepublik Deutschland (Anm. 2), S. 17.

61 Vgl. auch Gleß, Festschrift für Grünwald, S. 211. 


\section{bb) Gewäbrleistung einer "fairen Tatsachenfeststellung"}

Neben der - in den vorangegangenen Ausführungen in den Vordergrund gestellten - beweisrechtlichen Perspektive haben Beweisregeln weitere Zielsetzungen. So schützen sie etwa Grundrechte der von Beweisgewinnungsmaßnahmen betroffenen Personen ${ }^{62}$ bzw. die "integrity of the proceedings" aus der Sicht der Rechtsordnung, in deren Namen die strafrechtliche Sachentscheidung getroffen wird ${ }^{63}$. Dieser Aspekt der Beweisvorschriften wird zumeist unter dem Topos „Fairness der Tatsachenfeststellung" abgehandelt ${ }^{64}$.

Darüber, was „fair" ist, existieren in den europäischen Rechtsordnungen jedoch ebenfalls unterschiedliche Ansichten. Nach dem Modell des Grünbuchs soll ein "faires Verfahren" dadurch sichergestellt werden, dass die Verfahrensstandards und Eingriffsvoraussetzungen einer mitgliedstaatlichen Rechtsordnung, nämlich derjenigen des Erhebungsortes, eingehalten werden.

Um noch einmal das Fallbeispiel aufzugreifen: Im Rahmen des französischen Ermittlungsverfahrens gegen F (wegen des Verdachts des Betruges) ordnet der Untersuchungsrichter eine Überwachung des Telefonanschlusses im Privathaus des F an. Der Inhalt der aufgezeichneten Gespräche erhärtet den Verdacht. Das Amt für Betrugsbekämpfung (OLAF) möchte, dass die Staatsanwaltschaft Düsseldorf die aus der Telefonüberwachung gewonnenen Erkenntnisse auch in das Strafverfahren gegen D einführt. Der Verteidiger des $\mathrm{D}$ widerspricht mit der Begründung, dass die Anordnung der Telefonüberwachung zwar in Frankreich rechtmäßig gewesen sei, dass sie aber in Deutschland - mangels einer „Katalogtat" nach $\$ 100$ a StPO - nicht zulässig gewesen wäre.

Nach dem Kommissionskonzept des „freizügigen Beweises“ kann das Protokoll aus der Telefonüberwachung in Deutschland ohne weiteres

62 In der deutschen Dogmatik werden diese beiden unterschiedlichen Zielrichtungen durch die Kategorisierung als "unselbständige" (weil an einen vorangegangenen Verstoß gegen eine Beweisgewinnungsvorschrift anknüpfende) Beweisverwertungsverbote und „selbständige“ Beweisverbote gekennzeichnet; dazu etwa Dencker, Festschrift für Meyer-Goßner, S. 239, 244 f.

63 Zum Zusammenhang zwischen "integrity of the proceedings" und Rechtsschutz der Betroffenen Dencker, Festschrift für Meyer-Goßner, S. 245 f.

64 Für Deutschland vgl. etwa Eisenberg (Anm. 5), Rdn. 1050ff.; Rzepka, Zur Fairneß im deutschen Strafverfahren, 2000, S. 361 ff.; für England (unter besonderer Berücksichtigung der EMRK) Choo, Hearsay and Confrontations in Criminal Trials, S. 186ff.; für Frankreich Buisson, Encyclopédie Dalloz (März 1999), „Preuve“, Rdn. 122. 
verwertet werden. Der Einwand, dass in Deutschland diese Beweise nicht hätten erhoben werden dürfen, greift nicht durch. Die unterschiedlichen Wertungen der nationalen Gesetzgeber in der Abwägung von Strafverfolgungsinteressen gegenüber anderen schutzwürdigen Interessen (wie etwa der Familien- und Privatsphäre ${ }^{65}$ ) und die darauf gegründeten unterschiedlichen Voraussetzungen für Eingriffe (wie eine Überwachung des Telekommunikationsverkehrs) werden für die Frage der Beweisverwertung hinfällig ${ }^{66}$. Das Gericht verwertet einen Beweis, den es nach seiner eigenen Rechtsordnung weder hätte erheben noch hätte verwerten dürfen. An die Stelle des Gesetzgebers, der durch die Rechtsgemeinschaft, in deren Namen das Urteil gefällt wird, legitimiert ist, tritt insoweit ein fremder Gesetzgeber ${ }^{67}$.

Neben die Frage, ob unter diesen Umständen aus Sicht des Urteilsstaates noch eine "faire Tatsachenfeststellung" erfolgt, tritt ein weiterer Aspekt: In jedem Rechtssystem gibt es ein spezifisches System, das niedrigere Anforderungen bei der Gewinnung von Beweismitteln in einem Bereich durch höhere Anforderungen in einem anderen Bereich wieder ausgleicht. So wird beispielsweise das Fehlen eines enumerativen Straftatenkatalogs als Voraussetzung für die Anordnung einer Telefonüberwachung in Frankreich durch andere Verfahrensanforderungen kompensiert: Eine Telefonüberwachung kann nur nach Eröffnung einer gerichtlichen Voruntersuchung durch den Untersuchungsrichter, nicht aber durch die Staatsanwaltschaft oder gar durch die Polizei ${ }^{68}$ angeordnet werden ${ }^{69}$.

65 Die damit zusammenhängende Grundrechtsproblematik tritt noch deutlicher bei grenzüberschreitenden Maßnahmen hervor, wie sie etwa in dem geplanten Rahmenbeschluss über die Vollstreckung von Entscheidungen über die Sicherstellung von Vermögensgegenständen oder Beweismitteln in der EU (6980/02 COPEN 20) vorgesehen sind.

66 Auch greift hier der Vorschlag der Kommission zum Beweisausschluss nicht ein, nach dem ein Beweismittel, das rechtswidrig in einem Mitgliedstaat erlangt wurde, nach den Regeln des Erhebungsstaates von der Verwertung ausgeschlossen werden soll. Denn die Protokolle wurden ursprünglich rechtmäßig erlangt; die Bedenken ergeben sich aus der Verwertungssituation.

67 Grundsätzlich zur Bedeutung unterschiedlicher Eingriffsschwellen in den mitgliedstaatlichen Rechtsordnungen Nelles, ZStW 109 (1997), S. $749 f$.

68 Erkenntnisse aus präventiv-polizeilichen Telefonüberwachungen dürfen nicht als strafprozessuale Beweise verwertet werden; Hartwig, in: Gleß/Grote/Heine (Hrsg.), Justitielle Einbindung und Kontrolle von Europol, Bd. 2, 2001, S. 261.

69 Vgl. Art. 100 CPP; Matsopoulou, Les enquêtes de police, Paris 1996, S. $257 \mathrm{ff}$. 


\section{c) Zwischenergebnis}

Die wenigen Beispielfälle beleuchten nur schlaglichtartig die Probleme, die ein Beweistransfer zwischen unterschiedlich strukturierten (Beweis-) Rechtsordnungen aufwirft. Doch dies genügt, um zu erkennen, dass das Grünbuch-Modell nicht geeignet ist, die mit einem Beweistransfer verbundenen Probleme zu lösen. Denn es lässt einerseits grundlegende mit dem Beweistransfer verbundene Fragen offen und erhebt andererseits eine einzelne (weder notwendige noch hinreichende) Bedingung zum maßgeblichen Kriterium für die Zulässigkeit einer Beweisverwertung.

\section{Fazit und Ausblick}

Strafprozessuale Beweise sind nach ihrer Erhebung eben - anders als Wirtschaftswaren - keine fertigen Produkte, die ohne weiteren Bezug zu ihrer Erhebungssituation oder zu ihrer (antizipierten) Verwertungssituation in den „freien Warenverkehr" entlassen werden könnten. Bereits insoweit geht der Grünbuch-Vorschlag von falschen Prämissen aus. Das darauf gegründete Modell einer gegenseitigen Anerkennung vermag deshalb keine befriedigende Lösung für die Fragen des Beweistransfers bereitzuhalten.

Ausgangspunkt einer Lösung für die durch einen Beweistransfer verursachten Störungen muss die Funktion des strafprozessualen Beweises im nationalen Strafverfahren sein: seine - in allen nationalen Rechtsordnungen gleiche - Funktion als Grundlage für eine zuverlässige und faire Tatsachenfeststellung für eine strafrechtliche Sachentscheidung. Da diese Funktion in den mitgliedstaatlichen Verfahren - aufgrund der Unterschiede in der Ausgestaltung der Gerichtsorganisation und des strafrechtlichen Hauptverfahrens - auf verschiedene Weise sichergestellt wird, unterliegen Beweissammlung und -verwertung in den nationalen Verfahrensordnungen unterschiedlichen Beweisregeln. Ein tragfähiges Modell für einen EU-weiten Beweistransfer müsste die Verschiedenheit dieser nationalen Beweisregelungen - in ihrer spezifischen Funktion - berücksichtigen. Denn sie sind eben keine zufälligen Gegebenheiten, die durch das Postulat einer gegenseitigen Anerkennung ohne weiteres nivelliert werden könnten.

Eine Lösung für die Probleme des Beweistransfers könnte durch ein eigenes „europäisches Beweiszulassungsverfabren " herbeigeführt werden, das geeignet ist, für die einzelnen Systeme die Bedingungen sicherzustellen, die in der jeweiligen Rechtsordnung die Herstellung einer zuverlässigen Tatsachengrundlage in einem fairen Verfahren gewährleisten. 
Als Referenzsystem für die inhaltliche Ausgestaltung einer entsprechenden Beweiszulassungsordnung könnte auf die Vorgaben der Europäischen Menschenrechtskonvention (EMRK) zurückgegriffen werden. Dieser Weg erscheint nahe liegend, weil die EMRK geltendes Recht in den Mitgliedstaaten ist und dadurch bereits zu einer bemerkenswerten Annäherung der europäischen Strafverfahrensordnungen geführt hat. Eine solche Lösung dürfte deshalb von all denen begrüßt werden, die in den EMRK-Vorgaben einen gemeinsamen Mindestverfahrensstandard sehen ${ }^{70}$.

Doch wäre auch ein solches Modell nicht ohne Probleme: Ganz abgesehen von der Frage, ob durch die EMRK-Vorgaben nur ein Minimalstandard oder das wünschenswerte Niveau des Rechtsschutzes gewährleistet werden kann, erscheint aus zwei Gründen zweifelhaft, dass der bestehende EMRK-Acquis die notwendigen Anforderungen für alle (problematischen) Fälle des Beweistransfers bereithält: Zum ersten fehlen in der EMRK ausdrückliche Vorgaben über das strafprozessuale Beweisrecht. Dementsprechend haben die Straßburger Organe immer wieder betont, dass die Ausgestaltung der Beweisregelungen grundsätzlich alleinige Sache des nationalen Gesetzgebers ist ${ }^{71}$. Anerkannt ist aber, dass die Pflicht zur Gewährleistung bestimmter EMRK-Garantien - etwa des Rechts auf Konfrontation mit Belastungszeugen - eine bestimmte Beweisführung im nationalen Verfahren fordern bzw. ausschließen $\mathrm{kann}^{72}$; insofern existieren auch für das Beweisrecht bestimmte EMRK-Vorgaben ${ }^{73}$. Zum zweiten bestehen Bedenken gegen die Eignung der EMRK als transnationales Rechtsschutzsystem, weil die Straßburger Rechtsprechung in den zur Überprüfung vorgelegten Fällen bisher (nur) zur Sicherstellung von Einzelfallgerechtigkeit innerhalb eines nationalen Systems aufgerufen war. Dieser Ansatz führte zum einen zu einer kasuistischen Rechts(fort)bildung; zum anderen forderte diese Perspektive, dass der spezifische Zusammenhang innerhalb eines nationalen Systems in den Vordergrund gestellt wurde. Letzteres kommt vor allem in der "Ge-

70 Jung, JuS 2000, 419; Kühne, Strafprozeßrecht, 5. Aufl. 1999, Rdn. 43; Weigend, ZStW 105 (1993), S. 777 f; vgl. auch den Rückgriff auf die Vorgaben der EMRK durch die Vorschläge der „Corpus Juris“-Autorengruppe (Anm. 27).

71 Vgl. etwa Schenk v. Schweiz, EuGRZ 1988, 390; Teixeira de Castro v. Portugal, NStZ 1999, 47 m. Anm. Sommer; Khan v. Vereinigtes Königreich, JZ 2000, 993 m. Anm. Kïhne/Nash.

72 Vgl. etwa Schenk v. Schweiz, EuGRZ 1988, 390; dazu aus deutscher Sicht Kinzig, StV 1999, 288f.; Weigend, StV 2001, 64; aus französischer Sicht Crim. 5. Sept. 1994, B. 94.

73 Vgl. etwa Rzepka (Anm. 64), S. $361 \mathrm{ff}$ 
samtbetrachtungslehre" des EGMR zum Ausdruck ${ }^{74}$ : Danach wird ein Verstoß gegen ein in Art. 6 Abs. 3 EMRK verbürgtes Einzelrecht nur dann als Vertragsverletzung anerkannt, wenn dadurch das Verfahren im Einzelfall insgesamt als unfair betrachtet werden muss ${ }^{75}$. Wenn der Verstoß innerhalb des nationalen Systems wieder kompensiert wurde, liegt kein Vertragsverstoß vor. Diese Ausgleichsmöglichkeiten innerhalb eines nationalen Systems bestehen bei einem Beweistransfer aber gerade nicht. Um einen Ausgleich zwischen den Systemen herzustellen, bedürfte es einer Fortentwicklung der bestehenden Rechtsprechung, die - soweit möglich - Kompensationen zwischen verschiedenen Verfahrensordnungen berücksichtigen müsste.

Wer für diese Fortentwicklung zuständig sein sollte, bedarf ebenfalls der Klärung: Zum ersten wäre eine Ansiedlung der Entscheidungsgewalt über Fragen des Beweistransfers auf nationaler, zum zweiten auf europäischer Ebene denkbar, und zum dritten eine Kombination beider Ebenen, also eine regelmäßig bei den mitgliedstaatlichen Gerichten angesiedelte Entscheidungsbefugnis mit Vorlagepflicht an eine übergeordnete Instanz mit dem Ziel der Sicherung einer europaweit einheitlichen Rechtsentwicklung ${ }^{76}$.

Die Frage der EG-Kommission „Ist der Grundsatz, nach dem in einem Mitgliedstaat rechtmäßig erhobene Beweise vor den Gerichten der anderen Mitgliedstaaten zugelassen werden sollten, Ihrer Ansicht nach geeignet, in Bezug auf den Europäischen Staatsanwalt die Hindernisse aufgrund der unterschiedlichen Regelungen über die Zulässigkeit von Beweisen zu überwinden?" 77 muss jedenfalls mit „Nein." beantwortet werden.

74 Dieser Ansatz beruht auf der Prämisse des EGMR, dass die Einzelrechte des Art. 6 Abs. 3 EMRK nur unselbständige Ausprägungen des "fair trial"-Grundsatzes seien und ein Einzelverstoß daher im Gesamtverfahren kompensiert werden könne. Hintergrund dieser Interpretation ist das Bemühen des übergeordneten europäischen Gerichts, den Unterschieden der nationalen Verfahrensordnungen gerecht zu werden, die jeweils ganz spezifische Kompensationsmöglichkeiten für Verfahrensverstöße bereithalten können, welche bei isolierter Betrachtung als Konventionsverletzungen angesehen werden müssten; vgl. auch Kühne, StV 2001, 77.

75 Windisch v. Österreich, StV 1991, 193, Nr. 25; Asch v. Österreich, EuGRZ 1992, 474, Nr. 26; kritisch Kübne/Nash, JZ 2000, 997.

76 Vgl. dazu etwa die Zuständigkeitsverteilung zwischen (deutschen) nationalen Gerichten und dem EuGH nach Art. 35 EUV i.V.m. EuGH-Gesetz v. 6.8.1998, BGBl. I S. 2035; BGBI. I (1999), S. 728.

77 Grünbuch (Anm. 1), 6.3.4.1. 